\title{
Infecções odontogênicas complexas: Uma revisão de literatura
}

\author{
Complex odontogenic infections: A literature review \\ Infecciones odontogénicas complejas: Revisión de la literatura
}

Recebido: 29/06/2021 | Revisado: 09/07/2021 | Aceito: 14/07/2021 | Publicado: 24/07/2021

\author{
Eugênia Leal de Figueiredo \\ ORCID: https://orcid.org/0000-0001-5210-1389 \\ Universidade Federal de Pernambuco, Brasil \\ E-mail: eugeniafigueiredo@ hotmail.com \\ Eduarda Leal de Figueiredo \\ ORCID: https://orcid.org/0000-0002-2173-3487 \\ Universidade de Pernambuco, Brasil \\ E-mail: dudinhalf@hotmail.com \\ Carolina Pereira da Silva \\ ORCID: https://orcid.org/0000-0002-0058-3096 \\ Universidade Federal de Pernambuco, Brasil \\ E-mail: carolinapereeira@gmail.com \\ Maria Emanuella Letícia da Silva \\ ORCID: https://orcid.org/0000-0002-7159-1165 \\ Centro Universitário Brasileiro, Brasil \\ E-mail: emanuellaleticia20@gmail.com \\ Luana dos Santos Fonseca Peixoto \\ ORCID: https://orcid.org/0000-0001-7733-5157 \\ Universidade de Pernambuco, Brasil \\ E-mail: luanasfonseca6@gmail.com \\ Heitor Tavares de Araújo \\ ORCID: https://orcid.org/0000-0001-5906-3565 \\ Universidade de Pernambuco, Brasil \\ E-mail: heitortav@gmail.com \\ Jair Carneiro Leão \\ ORCID: https://orcid.org/0000-0001-8303-2291 \\ Universidade Federal de Pernambuco, Brasil \\ E-mail: jair.leao@ufpe.br \\ Alessandra de Albuquerque Tavares Carvalho \\ ORCID: https://orcid.org/0000-0003-0390-3611 \\ Universidade Federal de Pernambuco, Brasil \\ E-mail: alessandra.tcarvalho@ufpe.br
}

\begin{abstract}
Resumo
Infecções odontogênicas são comumente encontradas na região oral e maxilofacial, com isso, o manejo correto dessa condição se torna uma condição imprescindível para os cirurgiões maxilofaciais. Este estudo visa pontuar infecções complexas advindas de fonte odontogênica, analisando o conceito, diagnóstico e a forma de tratamento adequado a fim de contribuir com uma melhor evolução do quadro clínico. Realizou-se uma revisão literária através do cruzamento dos descritores selecionados em busca eletrônica entre os anos de 2006 e 2021, encontrados nos periódicos das bases de dados SCIELO, LILACS e MEDLINE. Entre as infecções complexas de origem odontogênica, pode-se citar Angina de Ludwig, Trombrose séptica do seio cavernoso, Mediastinite descente necrosante, Sinusite odontogênica, Abcesso cerebral e Fasceíte necrosante. Embora todas serem complicações diante da infecção e sintomatologia semelhante, cada uma tem características peculiares onde é possível diferencia-las. A conduta adotada diante essas Infecções é fator primordial para o sucesso do tratamento. Conclusão: O conhecimento de áreas anatômicas bem como a capacitação do profissional na prevenção, diagnóstico e, sobretudo, na resolução clínica, é essencial para amenizar a gravidade dessas infecções.
\end{abstract}

Palavras-chave: Angina de Ludwig; Infecção focal dentária; Mediastinite.

\begin{abstract}
Odontogenic infections are commonly found in the oral and maxillofacial region, therefore, the correct management of this condition becomes an essential condition for maxillofacial surgeons. This study aims to point out complex infections arising from odontogenic sources, analyzing the concept, diagnosis and form of appropriate treatment in order to contribute to a better evolution of the clinical picture. A literature review was carried out through the crossing of selected descriptors in electronic search between the years 2006 and 2021, found in the journals of the SCIELO, LILACS and MEDLINE databases. Among the complex infections of odontogenic origin, one can mention Ludwig's
\end{abstract}


Angina, Septic Cavernous Sinus Thrombrosis, Necrotizing Descending Mediastinitis, Odontogenic Sinusitis, Cerebral Abscess and Necrotizing Fasciitis. Although they are all complications in the face of infection and similar symptoms, each one has peculiar characteristics where it is possible to differentiate between them. The approach adopted in the face of these infections is a primordial factor for the success of the treatment. Conclusion: Knowledge of anatomical areas as well as professional training in prevention, diagnosis and, above all, in clinical resolution, is essential to alleviate the severity of these infections.

Keywords: Ludwig's Angina; Focal infection dental; Mediastinitis.

\section{Resumen}

Las infecciones odontogénicas se encuentran comúnmente en la región oral y maxilofacial, por lo que el manejo correcto de esta condición se convierte en una condición esencial para los cirujanos maxilofaciales. Este estudio tiene como objetivo señalar infecciones complejas derivadas de fuentes odontogénicas, analizando el concepto, diagnóstico y forma de tratamiento adecuado para contribuir a una mejor evolución del cuadro clínico. Se realizó una revisión de la literatura cruzando descriptores seleccionados en búsqueda electrónica entre los años 2006 y 2021, encontrados en las revistas de las bases de datos SCIELO, LILACS y MEDLINE. Entre las infecciones complejas de origen odontogénico, se pueden mencionar la Angina de Ludwig, Trombrosis Séptica del Seno Cavernoso, Mediastinitis Necrotizante Descendente, Sinusitis Odontogénica, Abceso Cerebral y Fascitis Necrotizante. Aunque todas son complicaciones ante la infección y síntomas similares, cada una tiene unas características peculiares donde es posible diferenciarlas. El abordaje adoptado ante estas infecciones es un factor primordial para el éxito del tratamiento. Conclusión: El conocimiento de las áreas anatómicas así como la formación profesional en prevención, diagnóstico y, sobre todo, en resolución clínica, es fundamental para paliar la gravedad de estas infecciones.

Palabras clave: Angina de Ludwig; Infección focal dental; Mediastinitis.

\section{Introdução}

As infecções odontogênicas apresentam uma das patologias mais comuns na região oral e maxilofacial, sendo caracterizadas como uma infecção dos alvéolos, mandíbulas ou rosto que se origina de um dente ou de suas estruturas de suporte, sendo uma das infecções mais frequentemente encontradas. Suas causas mais comuns são cárie dentária, obturações profundas ou falha no tratamento do canal radicular, pericoronarite e periodontites (Ogle, 2017; Katoumas et al., 2019).

A infecção começa localmente ao redor de um dente e pode permanecer localizada na região onde começou, ou pode se espalhar para áreas adjacentes ou distantes. O curso da infecção depende da virulência da bactéria, dos fatores de resistência do hospedeiro e da anatomia regional (Ogle, 2017). Dentre os sinais e sintomas relacionados a este quadro, o edema, dor no assoalho bucal, febre, disfagia, sialorréia, odor fétido, trismo e odontalgia são os mais comumente observados. Podem também ocorrer mudanças na fonação, aflição respiratória e cianose que refletem os sinais do comprometimento das vias aéreas (Camargos et al, 2016).

O curso da infecção depende da virulência da bactéria, dos fatores de resistência do hospedeiro e da anatomia regional. A sua gravidade pode variar de baixo grau, onde se tem infecções localizadas que requerem tratamento mínimo, até infecções graves como angina de Ludwig, trombose séptica do seio cavernoso, mediastinite, sinusite, obstrução das vias aéreas, abscesso cerebral e fasceíte necrosante, podendo até mesmo levar à morte. Por este motivo, o manejo desses cuidados se torna uma condição séria que os cirurgiões buco-maxilo-faciais precisam ter (Ogle, 2017; Katoumas et al., 2019).

Este estudo tem como objetivo pontuar infecções complexas advindas de fonte odontogênica, analisando o conceito, diagnóstico e a forma de tratamento adequado a fim de contribuir com uma melhor evolução do quadro clínico.

\section{Metodologia}

O presente estudo trata-se de uma revisão da literatura, desenvolvida por meio de fontes indexadas nas bases de dados do Scientific Eletronic Library Online (SCIELO), Literatura Latino Americana e do Caribe em Ciências da Saúde (LILACS) e Medical Literature Analysis and Retrival System Online (MEDLINE). Buscou-se por estudos publicados entre o período de 2006 e 2021, fazendo um cruzamento entre os descritores “Angina de Ludwig”, "Infecção focal dentária” e "Mediastinite”. Os 
artigos foram pesquisados nos idiomas português e inglês e a partir de sua análise na íntegra, foram selecionados e contribuíram para a análise descritiva deste trabalho.

\section{Resultados e Discussão}

\section{Angina de Ludwig}

A angina de Ludwig descrita em 1836 por Wilhelm Frederick Von Ludwig é considerada uma situação infectoinflamatória, de envolvimento polimicrobiano, que acomete áreas anatômicas submandibulares bilaterais, sublinguais e submentoniana (Barbosa et al, 2020). A progressão da infecção pode causar o envolvimento do espaço retrofaríngeo delimitado pela fáscia cervical profunda que inicia na base do crânio e se estende até o mediastino superior. Constitui uma entidade de difícil manejo devido à rápida progressão e à dificuldade na manutenção da via aérea pérvia e culmina em asfixia e morte em 8\%-10\% dos pacientes (Fellini et al, 2015).

Dessa forma, o estabelecimento de uma via aérea pérvia é a principal preocupação e pode necessitar de traqueostomia de urgência ou de uma intubação com fibra óptica por via nasal quando há suspeita do comprometimento da via aérea difícil (Fellini et al, 2015). Vale salientar, que o manejo inadequado pode romper o abscesso resultando na aspiração de material purulento com complicações pulmonares subsequentes. Se houver presença de edema, manipulação excessiva da via aérea não é aconselhada, pois pode causar sangramento ou laringoespasmo que necessitem de uma via aérea cirúrgica emergente e em muitos pacientes, a intubação oral não é possível (Fernandes et al, 2020).

\section{Trombose séptica do seio cavernoso}

O seio cavernoso é um sítio comum da ocorrência de trombose séptica. Apesar de ser uma ocorrência rara, quando acontece, é secundária a infecções de seios da face, órbitas, tonsilas, palato, dentes e seios esfenoidal e etmoidal. Possui características clínicas não específicas como: cefaleia, febre, toxemia, prostração, náusea, febre e o nível de consciência diminuem à proporção que há o avanço da infecção. Já a proptose, quemose e edema palpebral, provocados pela obstrução da veia oftálmica, são sinais considerados como tríade clássica (Houly et al, 2009; Ribeiro et al, 2015).

O diagnóstico é clínico e pode ser predito a partir de alterações das funções dos nervos cranianos que os cruzam (III, IV, V1 e V2 e VI), realizado por angiografia cerebral, tomografia ou ressonância magnética, com preferência para este último método diagnóstico em termos de custo-efetividade e segurança, pois consegue evidenciar os achados mais típicos desta condição, tais como: convexidade do seio cavernoso, falhas de enchimento, realce do seio cavernoso, dilatação da veia oftálmica superior e redução do lúmen carotídeo (Ribeiro et al, 2015).

Para realização do tratamento, a antibioticoterapia e anticoagulantes foi por um tempo vista como única forma de tratamento, mas a intervenção cirúrgica na área necrosada tem sua importância, uma vez que visa diminuir significativamente a população bacteriana no local, proporcionando um ambiente mais limpo com a diminuição de sequelas como: cegueira, parestesia, diplopia e complicações como a embolia pulmonar, meningite, abcesso cerebral, entre outras (Houly et al, 2009).

\section{Mediastinite descendente necrosante}

A mediastinite descendente necrosante ocorre normalmente após infecções orofaríngeas e cervicofaciais. Apesar de ser uma forma mais rara, também pode se desenvolver após infecção odontogênica, tendo uma alta taxa de mortalidade com aproximadamente $40 \%$ (Conceição et al, 2019). Esta infecção alcança o mediastino pelo sinergismo entre força da gravidade, movimentos respiratórios e pressão intratorácica negativa, criando um curso da coleção purulenta determinado pelos espaços e planos fasciais e cervicais profundos. Por esses espaços anatômicos serem pobremente vascularizado, é formado uma via 
facilitadora para proliferação de microrganismos anaeróbios, componentes de parte da flora dessa enfermidade (Silva et al, 2017).

Considerada uma condição grave, caracterizada pela dor, aumento do volume do pescoço, rigidez da musculatura cervical, enfisema subcutâneo, disfagia decorrente da compressão do esôfago pela coleção purulenta, dispneia e dor torácica por pneumonite aspirativa, o paciente termina por ficar debilitado sistematicamente, exigindo um rápido diagnóstico para realização do tratamento (Silva et al, 2017).

O tratamento exige uma drenagem cirúrgica precoce em associação a antibioterapia adequada, baseado no controle adequado dos distúrbios hidroeletrolíticos e das alterações respiratórias, as quais podem exigir intubação ou traqueostomia (Moreira et al, 2017; Silva et al, 2017). Penicilinas e cefalosporinas são os antibióticos de escolha, tendo também indicação da gentamicina. A associação de penicilina com metronidazol é bastante empregada nos casos de infecções severas com presença de coleção purulenta, pelo fato de o metronidazol combater com eficácia as bactérias anaeróbias e havendo constatação de infecção por pseudomonas, recomenda-se a utilização de meropenem (Conceição et al, 2019; Silva et al, 2017).

\section{Sinusite odontogênica}

A Sinusite maxilar crônica de origem dentária é uma doença cuja prevalência é muitas vezes subestimada. A causa mais comum é o granuloma apical seguido da periodontite, especialmente do primeiro molar e é mais comum em adultos do que em crianças (Franche et al., 2006). O diagnóstico da sinusite odontogênica envolve o uso de exames por imagem que pode fornecer informações valiosas no diagnóstico (Lima et al., 2017). Avanços recentes na área de imagem odontológica indicam a utilização de imagens computadorizadas do tipo Cone-Beam, devido à qualidade da imagem e redução das doses de radiação ao paciente (Conto et al., 2013).

Os sintomas são semelhantes a sinusite não odontogênica, como obstrução ou congestão nasal, dor ou pressão na face e dores de cabeça. No entanto, quando a origem é odontogênica, geralmente, estes sintomas manifestam-se em apenas um lado da face. Outros sintomas como dor nos olhos, gotejamento pós-nasal, mau odor e dor de origem dentária também foram relatados (Lima et al., 2017). No tratamento da sinusite odontogênica é necessário atuar sobre a causa do dente e sobre a própria doença. Só então é possível eliminar a infecção existente e prevenir recorrências ou complicações. A combinação de terapêutica farmacológica e cirúrgica geralmente é necessária para o tratamento da sinusite odontogênica (Conto et al., 2013).

\section{Abcesso cerebral}

O abscesso cerebral se constitui em uma complicação grave, com risco de vida (Segura et al., 2015; Correia et al., 2018), caracterizado por uma área localizada de pus intracerebral (Fica et al., 2006; Correia et al., 2018). É uma patologia pouco frequente, mas com uma evolução prolongada que ocorre normalmente em homens após traumatismo ou cirurgia craniana, podendo ser originado também de infecções pericranianas ou disseminado por via hematológica. São diversos os agentes etiológicos, com dominância do Staphylococcus aureus, Strepcoccus sp e Haemophylos influenzae. Ainda, o Peptostreptococcus e Streptococcus são particularmente comuns em abscessos cerebrais causados por contiguidade (Correia et al., 2018).

O diagnóstico diferencial é amplo e a suspeita clínica deve ser precoce para se obter tratamento multidisciplinar em tempo hábil que garanta um bom prognóstico (Fica et al., 2006). As lesões têm início com área de cerebrite localizada no parênquima cerebral e evoluem para coleções de pus limitadas por cápsula. Podem se apresentar clinicamente em quatro síndromes básicas: expansão de massa focal, hipertensão intracraniana, destruição difusa ou déficit neurológico focal. Os sintomas mais comuns são: cefaleia, alteração do sensório, náusea e/ou vômito e febre alta (Correia et al., 2018). A tomografia computadorizada (TC) de crânio evidencia, na fase inicial da doença, uma lesão hipodensa, indefinida que, após a 
administração de contraste, apresenta um realce anular; quando é envolvido por cápsula visualiza-se um centro hipodenso, demonstrando a necrose central com liquefação, limitada por anel hiperdenso (a cápsula) - Em ambas fases a TC mostra um edema circundante à lesão (Correia et al., 2018).

Pode levar a sérios déficits, ou morte se mal diagnosticado ou manejado de forma incorreta. Com isso, seu tratamento não é restritivo e cada caso deve ser individualizado. Recomenda-se a instituição de antibioticoterapia imediata, com predileção à associação de: cefotaxima/ceftriaxone/ceftazidima, vancomicina e metronidazol. Além disso, a maioria dos abscessos necessita de abordagem cirúrgica, sendo necessário drenar o abscesso através de craniotomia. Quando adequadamente realizada, produz uma melhora clínica imediata e estabiliza o paciente hemodinamicamente. A escolha da técnica cirúrgica deve ser específica para cada paciente (Correia et al., 2018).

\section{Fasceite Necrozante}

A fasceíte necrosante da cabeça e pescoço é uma rara e potencialmente fatal infecção bacteriana do tecido mole, que acomete principalmente indivíduos adultos e idosos, sem predileção por sexo (Medeiros Júnior et al., 2011). A maioria dos casos tem origem odontogênica, envolvendo abscessos dentários e doença periodontal crônica, ou faríngea; evoluindo com extensa necrose e formação gasosa no tecido subcutâneo e fascial subjacente, com elevado índice de mortalidade (aproximadamente 40\%) (Medeiros Junior et al., 2011; Camino Junior et al., 2014).

Os exames imaginológicos são fundamentais para caracterizar os limites topográficos da infecção e o diagnóstico diferencial deve ser feito principalmente com celulite ou erisipela em seu estágio inicial. $\mathrm{O}$ tratamento bem sucedido envolve o diagnóstico precoce, desbridamento cirúrgico radical de todo o tecido necrótico, pois existe uma morbidade potencialmente elevada, combinada com antibioticoterapia parenteral de amplo espectro e medidas gerais de suporte agressivas (Medeiros Junior et al., 2011).

\section{Considerações Finais}

As infecções odontogênicas apresentam uma das patologias mais comuns na região oral e maxilofacial, as quais podem evoluir para áreas adjacentes levando a quadros de alta morbidade e mortalidade. O conhecimento de áreas anatômicas, bem como a capacitação do profissional na prevenção, diagnóstico e, sobretudo, na resolução clínica, torna-se importante para amenizar a gravidade e como consequência o risco de vida advindo destas infecções.

\section{Referências}

Barbosa, L. M., Negreiros, J. H. C. N., de Morais Neves, L. E., Pinto, P. S., de Oliveira, L. M. L., Silva, M. L. D. A. C., \& Laureano Filho, J. R. (2020). Angina de Ludwig associada a complicações mediastinais: Relato de caso. Research, Society and Development, 9(9), e764997824-e764997824.

Camino Junior, R., Naclerio-Homem, M. G., Cabral, L. M., \& Luz, J. G. C. (2014). Cervical necrotizing fasciitis of odontogenic origin in a diabetic patient complicated by substance abuse. Brazilian dental journal, 25(1), 69-72.

Conto, F., De Bona, M., Rui, G., Rovani, G., Rhoden, R., \& Ericson Flores, M. (2013). Sinusitis Maxilar de Origen Odontogénica: Diagnóstico y Tratamiento Quirúrgico. International journal of odontostomatology, 7(3), 421-426.

Conceição, P. F. G., de Jesus Moureira, P. A., \& Ribeiro, P. M. L. (2019). Mediastinite descendente necrosante pós-angina de Ludwig: relato de caso. Revista de Ciências Médicas e Biológicas, 18(3), 425-428.

Correia, F. C. S., Gonçalves, P. P. F., Martins, A. L., Tauil, V. M. A., Marinho, M. F., \& Reis, C. H. M. (2018). Abscesso Cerebral Secundário À Rinossinusite Bacteriana: Um Relato De Caso. Revista Saber Digital, 10(2), 11-19.

Fellini, R. T., Volquind, D., Schnor, O. H., Angeletti, M. G., \& de Souza, O. E. (2017). Manejo da via aérea na angina de Ludwig-um desafio: relato de caso. Brazilian Journal of Anesthesiology, 67(6), 637-640.

Fernandes, S. L., de Sousa Ferreira, L. P., de Oliveira, M. A., Fernandes, G. C., Neto, V. T., Santana, T. M., \& Moretto, M. J. (2020). Complicações relativas às infecções odontogênicas: Angina de Ludwig. Journal of Multidisciplinary Dentistry, 10(1), 46-51. 
Research, Society and Development, v. 10, n. 9, e20310917668, 2021

(CC BY 4.0) | ISSN 2525-3409 | DOI: http://dx.doi.org/10.33448/rsd-v10i9.17668

Fica, A., Bustos, P., \& Miranda, G. (2006). Absceso cerebral: A propósito de una serie de 30 casos. Revista chilena de infectología, 23(2), 140-149.

Franche, G. L., Krumenauer, R. C. P., Böhme, E. S., Mezarri, A., \& Wiebbeling, A. M. D. (2006). Sinusite odondogénica. RGO Porto Alegre, 54(2), 175-177.

Houly, L. R., Souza, M. M., MIKAMI, J. R., \& BARROS, H. P. (2020). Trombose séptica do seio cavernoso de origem odontogênica: relato de caso.

Katoumas, K., Anterriotis, D., Fyrgiola, M., Lianou, V., Triantafylou, D., \& Dimopoulos, I. (2019). Epidemiological analysis of management of severe odontogenic infections before referral to the emergency department. Journal of Cranio-Maxillofacial Surgery, 47(8), 1292-1299.

Lima, C. O., Devito, K. L., Vasconcelos, L. R. B., do Prado, M., \& Campos, C. N. (2017). Sinusite odontogênica: uma revisão de literatura. Revista Brasileira de Odontologia, 74(1), 40.

Medeiros Júnior, R., Melo, A. D. R., Oliveira, H. F. L. D., Cardoso, S. M. O., \& Lago, C. A. P. D. (2011). Fasceíte necrosante cérvico-torácica facial odontogênica. Brazilian Journal of Otorhinolaryngology, 77, 805-805.

Moreira, C., Melo, P., Ribeiro, C., \& Barros, E. (2016). Mediastinite descendente necrosante secundária a abcesso retrofaríngeo num adulto. Revista Portuguesa de Otorrinolaringologia e Cirurgia de Cabeça e Pescoço, 54(4), 265-269.

Ogle, O. E. (2017). Odontogenic Infections. Dental Clinics of North America, 61(2), 235-252.

Ribeiro, M. I. B., Guiotti, M. G., Correa, D. S. A., Rebello, L. C., \& Dias, R. M. (2015). Tromboflebite séptica de seio cavernoso com espessamento meníngeo de base de crânio. Comunicação em Ciências da Saúde, 26(01/02).

Segura, U., Neves, Z., Valente, A., Pacheco, M. H., \& Malhado, J. A. (2015). Abcesso cerebral num doente com Rendu-Osler-Weber. Revista da Sociedade Brasileira de Clinica Médica, 57-60.

Silva, K. T., Junior, E. A. G., Magro-Érnica, N., Griza, G. L., \& Tomazi, F. H. S. (2017). Mediastinite necrosante descendente após exodontia de terceiros molares. Revista da Faculdade de Ciências Médicas de Sorocaba, 19(4), 224-226.

Yuvaraj, V. (2016). Maxillofacial infections of odontogenic origin: epidemiological, microbiological and therapeutic factors in an Indian population. Indian Journal of Otolaryngology and Head \& Neck Surgery, 68(4), 396-399. 